\title{
Single-level lumbar pyogenic spondylodiscitis treated with mini-open anterior debridement and fusion in combination with posterior percutaneous fixation via a modified anterior lumbar interbody fusion approach
}

\author{
Yang Lin, MD, PhD, Feng Li, MD, PhD, Wenjian Chen, MD, PhD, Heng Zeng, MD, PhD, \\ Anmin Chen, MD, PhD, and Wei Xiong, MD, PhD \\ Department of Orthopedics, Tongji Hospital of Tongji Medical College, Huazhong University of Science and Technology, Wuhan, \\ P. R. China
}

OBJECT This study evaluated the efficacy and safety of mini-open anterior debridement and lumbar interbody fusion in combination with posterior percutaneous fixation for single-level lumbar pyogenic spondylodiscitis.

METHODS This is a retrospective study. Twenty-two patients with single-level lumbar pyogenic spondylodiscitis underwent mini-open anterior debridement and lumbar interbody fusion in combination with posterior percutaneous fixation via a modified anterior lumbar interbody fusion (ALIF) approach. Patients underwent follow-up for 24 to 38 months. Clinical data, etiological examinations, operative time, intraoperative blood loss, American Spinal Injury Association (ASIA) grade, Japanese Orthopaedic Association (JOA) lumbar function score, visual analog scale (VAS) score, Oswestry Disability Index (ODI), postoperative complications, and the bony fusion rate were recorded.

RESULTS The mean operative time was $181.1 \pm 22.6$ minutes (range 155-240 minutes). The mean intraoperative blood loss was $173.2 \pm 70.1 \mathrm{ml}$ (range 100-400 ml). Infection was found in lumbar vertebrae L2-3, L3-4, and L4-5 in 2, 6, and 14 patients, respectively. Bacterial cultures were positive in 15 patients, including 4 with Staphylococcus aureus, 6 with Staphylococcus epidermidis, 4 with Streptococcus, and 1 with Escherichia coli. Postoperative complications included urinary retention, constipation, and numbness in the thigh in 5, 3, and 2 patients, respectively. Compared with before surgery, the VAS scores and ODI were significantly lower at the final follow-up, the JOA scores were significantly higher, and the ASIA grades had improved. All patients achieved good intervertebral bony fusion.

CONCLUSIONS Mini-open anterior debridement and lumbar interbody fusion in combination with posterior percutaneous fixation via a modified ALIF approach results in little surgical trauma and intraoperative blood loss, acceptable postoperative complications, and is effective and safe for the treatment of single-level lumbar pyogenic spondylodiscitis. This approach could be an alternative to the conventional open surgery.

http://thejns.org/doi/abs/10.3171/2015.5.SPINE14876

KEY WORDS lumbar pyogenic spondylodiscitis; mini-open; percutaneous fixation; anterior lumbar interbody fusion; curative effect; complication; infection

$\mathrm{L}$ UMBAR pyogenic spondylodiscitis commonly results from primary hematogenous infection ${ }^{7}$ or occurs after lumbar vertebral surgery or invasive procedures. ${ }^{6}$ Conservative treatment is preferred, ${ }^{3,4}$ but surgery is required when conservative treatment fails or in cases of epidural abscess, spinal deformity, or neurological deficit symptoms. ${ }^{23}$ The purpose of surgical treatment is to debride the lesion, correct the spinal deformity, and decompress the nerve.
Currently, anterior debridement and fusion in combination with posterior pedicle screw fixation is widely used for the treatment of pyogenic spondylodiscitis. ${ }^{8,21,22}$ This approach can result in thorough debridement and stable internal fixation, improving the rates of inflammation resolution and bony fusion. ${ }^{8}$ However, conventional open surgery is associated with several disadvantages, including large surgical trauma, severe intraoperative blood loss, high postoperative complication rates, and a slow recov-

ABBREVIATIONS ALIF = anterior lumbar interbody fusion; $A S I A=$ American Spinal Injury Association; $C R P=C$-reactive protein; ESR = erythrocyte sedimentation rate; $\mathrm{JOA}=$ Japanese Orthopaedic Association; ODI = Oswestry disability index; VAS = visual analog scale; $W B C=$ white blood cell count.

SUBMITTED August 24, 2014. ACCEPTED May 19, 2015.

INCLUDE WHEN CITING Published online September 4, 2015; DOI: 10.3171/2015.5.SPINE14876. 
ery time. Therefore, mini-open methods are required to reduce complications and improve clinical outcomes.

In the present study, we performed mini-open anterior debridement and lumbar interbody fusion in combination with posterior percutaneous fixation via a modified anterior lumbar interbody fusion (ALIF) approach on 22 patients with single-level lumbar pyogenic spondylodiscitis between March 2008 and June 2010 in order to investigate the efficacy and safety of this mini-open surgical approach.

\section{Methods \\ Patients}

This is a retrospective study. The institutional review board of Tongji Hospital of Tongji Medical College approved this study, and all patients gave their informed consent. This study adheres to the principles set forth in the World Medical Association's Declaration of Helsinki. The study included 22 patients (13 men and 9 women) with single-level lumbar pyogenic spondylodiscitis who underwent mini-open anterior debridement and lumbar interbody fusion in combination with posterior percutaneous fixation at the Department of Orthopedics between March 2008 and June 2010. These patients had been given systemic antibiotics, which did not relieve their symptoms. The indications for surgery included the symptoms of nerve damage, epidural abscess, and spinal instability observed on imaging. The average age of these patients was $54.7 \pm 8.0$ years (range $43-71$ years; Table 1 ).

Preoperative examinations included routine blood, erythrocyte sedimentation rate (ESR), C-reactive protein (CRP), blood culture, tuberculosis antibody test, and TSPOT.TB test (Oxford Immunotec). Radiological studies consisted of lumbar posteroanterior and flexion-extension radiographs, CT with 3D reconstruction, and MRI. All patients were diagnosed with lumbar pyogenic spondylodiscitis based on clinical presentations such as severe deep lumbar pain and fever, laboratory examinations (elevated white blood cell counts [WBC], ESR, and CRP), and radiological imaging, especially MRI, that showed signs of intervertebral infection. ${ }^{13}$ Tuberculosis and tumors were excluded by laboratory tests and radiological imaging. Single-level lumbar pyogenic spondylodiscitis was found in the L2-3 (2 patients), L3-4 (6 patients), or L4-5 vertebrae (14 patients). Eight patients had nerve damage symptoms, 4 had epidural abscess, and 10 exhibited spinal instability with loss of intervertebral space height. Before surgical treatment, all patients received the systemic administration of antibiotics, but the symptoms were not relieved.

\section{Surgical Procedure}

Under C-arm fluoroscopy, patients were placed prone, and 2 pedicle screws were implanted in the proximal and distal vertebrae of the affected segment, respectively. If the lesions affected the screw implantation, fixation was extended 1 segment further. Then, the patients were turned to the right lateral position, and the incision site and surface markers were confirmed by $\mathrm{C}$-arm fluoroscopy. A mini-open horizontal incision was made in the left lumbocostal region at the intervertebral level of the affected segment. A modified ALIF approach was used to expose the affected intervertebral space. The external oblique, internal oblique, and transverse abdominal muscles were dissected in the direction of their fiber orientation. At the retroperitoneal space, the psoas muscle was exposed after pushing the retroperitoneal fat forward. The affected intervertebral space was exposed by bluntly dissecting the psoas muscle at about $5 \mathrm{~mm}$ behind the anterior edge of the psoas major muscle along the direction of its fiber orientation. A working channel was created using a miniopen Medtronic EndoRing, and the affected intervertebral space was clearly visualized by the surgeon (Fig. 1).

After the annulus was cut open, the pus and tissues in the intervertebral space were collected for bacterial culture, drug sensitivity testing, and pathological examination. All visible necrotic tissues, nucleus pulposus, endplate cartilage, sequestrum, and pus were thoroughly scraped, and the intervertebral space was extensively rinsed with $3 \%$ hydrogen peroxide, povidone iodine saline, and normal saline. The sterile surgical instruments were replaced, and an autologous iliac bone strut was removed carefully to avoid fracture and nerve injury, trimmed to an appropriate size, and implanted into the intervertebral space structurally. The length of the tricortical allograft strut was adapted to the height of intervertebral space, and the depth was fit to the width of the anterior third of the intervertebral space. After the grafted bone was compressed firmly, 1 irrigation tube and 1 drainage tube were placed into the intervertebral space, and another drainage tube was placed extraperitoneally. Intraoperative neurophysiological monitoring was used to monitor evoked potentials and electromyography in all cases, for both the anterior and posterior approaches. No severe monitoring findings occurred that affected the surgical procedure.

\section{Perioperative Management}

Patients received the intravenous administration of antibiotics after diagnosis, and the antibiotics were discontinued 3 days before surgery to improve the positive bacterial culture rate.,4,9 Postoperative broad-spectrum antibiotics and anti-gram-positive antibiotics were empirically used, and antibiotics were adjusted based on the results of the bacterial culture and drug sensitivity tests. Antibiotics were continuously used until the symptoms and signs disappeared and ESR and CRP levels returned to normal. ${ }^{17}$ For patients with a negative bacterial culture result, antibiotics were empirically used. The postoperative administration of gentamicin in saline into the lesion area via the irrigation tube continued for at least 3 weeks. The irrigation and drainage tubes were pulled out after 3 consecutive negative results of the bacterial cultures of the drainage liquids. Patients were instructed to exercise their lower limbs and back muscles in bed during the period of postoperative bed rest. When the symptoms and signs were relieved, patients were allowed to ambulate under the protection of a waist belt.

\section{Therapeutic Evaluation}

Patient symptoms and signs were evaluated after surgery. All patients underwent lumbar radiography at 3, 6, 
TABLE 1. Clinical characteristics and surgical parameters of 22 patients with lumbar pyogenic spondylodiscitis

\begin{tabular}{|c|c|c|c|c|c|c|c|c|c|}
\hline $\begin{array}{c}\text { Case } \\
\text { No. }\end{array}$ & Sex & $\begin{array}{l}\text { Age } \\
\text { (yrs) }\end{array}$ & Segment & Cause & $\begin{array}{l}\text { Course } \\
\text { (wks) }\end{array}$ & Bacterial Culture & $\begin{array}{c}\text { Surgery } \\
\text { (mins) }\end{array}$ & $\begin{array}{l}\text { Blood Loss } \\
\quad(\mathrm{ml})\end{array}$ & Complications \\
\hline 1 & M & 51 & L4-5 & Ozone ablation & 6 & Staphylococcus epidermidis & 180 & 180 & NA \\
\hline 2 & $\mathrm{~F}$ & 45 & L4-5 & Laser ablation & 5 & - & 210 & 400 & Urinary retention \\
\hline 3 & $\mathrm{~F}$ & 58 & L3-4 & Radiofrequency ablation & 8 & Streptococcus & 175 & 140 & NA \\
\hline 4 & $\mathrm{~F}$ & 61 & L3-4 & NA & 10 & - & 160 & 100 & NA \\
\hline 5 & M & 50 & L4-5 & Cutting-aspiration & 3 & Staphylococcus epidermidis & 155 & 150 & Urinary retention \\
\hline 6 & M & 48 & L4-5 & Radiofrequency ablation & 7 & Escherichia coli & 160 & 170 & NA \\
\hline 7 & $\mathrm{~F}$ & 44 & L3-4 & Cutting-aspiration & 5 & - & 185 & 160 & Constipation \\
\hline 8 & M & 52 & L4-5 & Ozone ablation & 11 & Staphylococcus epidermidis & 170 & 180 & NA \\
\hline 9 & M & 62 & L3-4 & NA & 8 & Staphylococcus aureus & 165 & 150 & Numbness in thigh \\
\hline 10 & $\mathrm{~F}$ & 60 & L4-5 & Laser ablation & 4 & Staphylococcus epidermidis & 170 & 170 & NA \\
\hline 11 & $\mathrm{~F}$ & 67 & L4-5 & NA & 5 & Staphylococcus aureus & 240 & 150 & Constipation \\
\hline 12 & M & 49 & L4-5 & Chemonucleolysis & 8 & - & 165 & 140 & Urinary retention \\
\hline 13 & M & 48 & L4-5 & Cutting-aspiration & 4 & Streptococcus & 220 & 180 & Numbness in thigh \\
\hline 14 & $\mathrm{~F}$ & 65 & L2-3 & NA & 12 & - & 160 & 210 & NA \\
\hline 15 & M & 50 & L4-5 & Radiofrequency ablation & 9 & Staphylococcus epidermidis & 205 & 350 & Urinary retention \\
\hline 16 & M & 51 & L4-5 & Cutting-aspiration & 5 & - & 170 & 120 & NA \\
\hline 17 & M & 71 & L3-4 & NA & 6 & Streptococcus & 180 & 170 & NA \\
\hline 18 & $\mathrm{~F}$ & 53 & L4-5 & Ozone ablation & 7 & Staphylococcus epidermidis & 200 & 150 & NA \\
\hline 19 & M & 59 & L2-3 & NA & 9 & Staphylococcus aureus & 170 & 120 & Constipation \\
\hline 20 & M & 43 & L3-4 & NA & 3 & Streptococcus & 165 & 140 & NA \\
\hline 21 & $\mathrm{~F}$ & 52 & L4-5 & Radiofrequency ablation & 8 & Staphylococcus aureus & 205 & 150 & Urinary retention \\
\hline 22 & $M$ & 65 & L4-5 & NA & 6 & - & 175 & 130 & NA \\
\hline
\end{tabular}

$\mathrm{NA}=$ not applicable.

and 12 months and CT scanning at 12 months after surgery to confirm intervertebral fusion. Routine blood tests, ESR, and CRP were examined weekly until the results returned to normal. Preoperative and postoperative spinal cord injury, pain, and disability were evaluated using the American Spinal Injury Association (ASIA) impairment classification, ${ }^{11}$ Japanese Orthopaedic Association (JOA) lumbar function score ${ }^{19}$ visual analog scale (VAS) score, ${ }^{12}$ and Oswestry Disability Index (ODI). ${ }^{5}$ Postoperative complications were recorded during the follow-up period.

\section{Statistical Analysis}

Statistical analyses were performed using SPSS 16.0 software (SPSS Inc.). The paired t-test was used to assess the difference between the preoperative and postoperative VAS scores and ODIs. Here, $\mathrm{p}$ values $<0.05$ were considered statistically significant.

\section{Results}

A summary of the clinical characteristics and surgical parameters of the 22 patients with lumbar pyogenic spondylodiscitis is detailed in Table 1. The mean operative time was $181.1 \pm 22.6$ minutes (range 155-240 minutes), and the mean intraoperative blood loss was $173.2 \pm 70.1$ $\mathrm{ml}$ (range 100-400 $\mathrm{ml}$ ). During the operation, soft tissue swelling was observed around the affected segment. Annular disruption and paraspinal abscess were seen in some patients. The intervertebral space was filled with necrotic nucleus pulposus, endplate cartilage, and sequestrum. The endplate cartilage was fragile and easily scraped off.

Of the 22 patients, preoperative positive blood bacterial culture results were found in 7 patients. Positive operative tissue bacterial culture results were found in 15 patients, including 4 patients with Staphylococcus aureus, 6 with

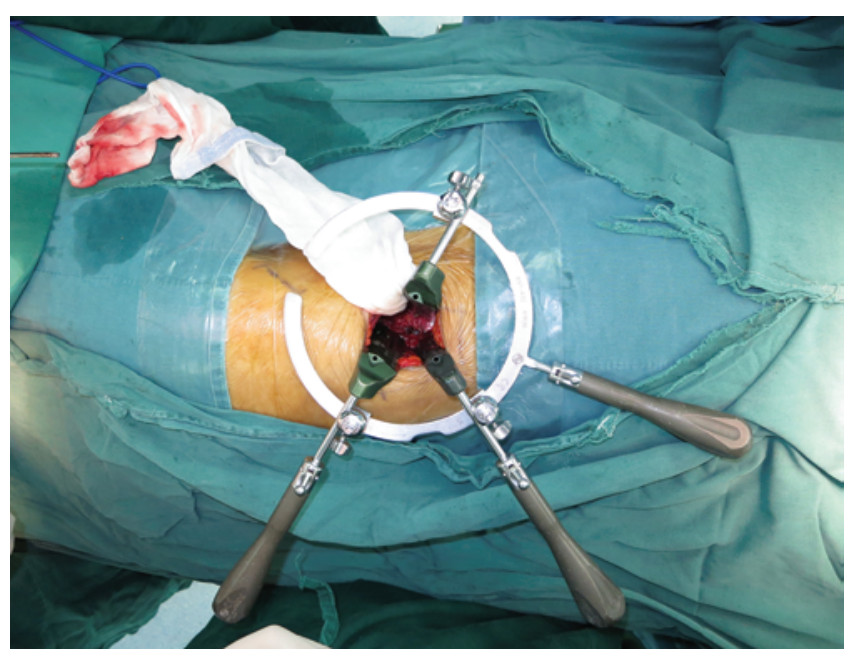

FIG. 1. Photograph showing the establishment of anterior mini-open working channel. Figure is available in color online only. 
Staphylococcus epidermidis, 4 with Streptococcus, and one with Escherichia coli (Table 1). All 7 patients with preoperative positive blood bacterial culture results had positive operative bacterial culture results. The remaining 7 patients had negative bacterial culture results. Postoperative pathological examination confirmed the diagnosis of pyogenic spondylodiscitis.

For all patients, symptoms such as back pain were obviously relieved immediately after surgery. The WBC, ESR, and CRP decreased gradually, and all were normal at the 3-month follow-up after surgery (Table 2). All surgical incisions achieved primary healing. Perioperative complications included urinary retention (5 patients), constipation (3 patients), and numbness in the thigh (2 patients). Urinary retention and constipation were transient and relieved after symptomatic treatment. Numbness in the thigh ipsilateral to the surgical side was caused by intraoperative traction of the lumbar plexus and had disappeared by the 3-months follow-up. No other complications such as dural rupture, nerve root injury, or pulmonary embolism occurred. No abscess or fistula was observed after the removal of irrigation and drainage tubes.

All patients underwent follow-up for $30.7 \pm 4.0$ months (range 24-38 months). The lumbar radiographs and CT scans showed that good intervertebral bony fusion was achieved 12 months after surgery in all patients (Fig. 2). No internal fixation loosening or breakage occurred. At the final follow-up, the mean JOA scores $(25.9 \pm 1.6)$ were significantly higher than they had been preoperatively
$(9.1 \pm 2.2 ; \mathrm{p}<0.001)$. The mean VAS scores $(0.6 \pm 0.7)$ were significantly lower than they were preoperatively (7.5 $\pm 0.8 ; \mathrm{p}<0.001)$. The ODIs at final follow-up $(14.7 \% \pm$ $5.7 \%$ ) were also significantly lower than the preoperative scores $(81.5 \% \pm 5.8 \% ; \mathrm{p}<0.001)$. The ASIA grades had improved at the final follow-up compared with the preoperative grades (Table 3). No recurrence was found at the final follow-up.

\section{Discussion}

Several surgical approaches have been applied to treat lumbar pyogenic spondylodiscitis, including posterior, ${ }^{14}$ anterior, ${ }^{25}$ and a combination of the anterior and posterior approaches. ${ }^{8,21,22}$ For the posterior approach, pedicle screws can provide stable 3-column spine fixation, but it is difficult to thoroughly debride the affected lesions and thus there is a high risk of the spread of bacteria to the screws and muscles posterior to the vertebrae. For the anterior approach, the intervertebral lesions can be completely removed under direct vision, ${ }^{25}$ thus reducing surgical interference with the spinal canal and avoiding bacterial spread. However, anterior fixation does not provide as strong of support as posterior fixation. Because of these considerations, anterior debridement in combination with posterior fixation is widely used for the treatment of lumbar pyogenic spondylodiscitis., ${ }^{8,21,22}$ Yet, conventional open surgery using this approach is associated with disadvantages such as a large incision, excessive tissue damage,

TABLE 2. Preoperative and 3-month postoperative follow-up CRP, ESR, and WBC

\begin{tabular}{|c|c|c|c|c|c|c|}
\hline \multirow[b]{2}{*}{ Case No. } & \multicolumn{2}{|c|}{ CRP (mg/L) } & \multicolumn{2}{|c|}{$\mathrm{ESR}(\mathrm{mm} / \mathrm{hr})$} & \multicolumn{2}{|c|}{$W B C \times 10^{9} / L$} \\
\hline & Preop & 3-Mo Follow-Up & Preop & 3-Mo Follow-Up & Preop & 3-Mo Follow-Up \\
\hline 1 & 95.3 & 3.2 & 52 & 3 & 10.3 & 6.6 \\
\hline 2 & 142.2 & 4.1 & 80 & 15 & 18.2 & 4.5 \\
\hline 3 & 113.6 & 6.8 & 66 & 11 & 11.8 & 6.9 \\
\hline 4 & 280.6 & 2.9 & 78 & 7 & 15.6 & 5.6 \\
\hline 5 & 340.5 & 6.0 & 95 & 10 & 13.1 & 4.2 \\
\hline 6 & 149.4 & 2.5 & 103 & 1 & 18.3 & 3.7 \\
\hline 7 & 140.5 & 8.1 & 77 & 5 & 14.3 & 6.8 \\
\hline 8 & 201.4 & 4.4 & 69 & 7 & 15.2 & 8.2 \\
\hline 9 & 136.7 & 4.8 & 73 & 12 & 12.0 & 4.6 \\
\hline 10 & 126.1 & 9.6 & 71 & 8 & 12.1 & 5.8 \\
\hline 11 & 88.3 & 3.6 & 49 & 14 & 10.9 & 4.3 \\
\hline 12 & 110.3 & 12.4 & 84 & 10 & 9.4 & 7.7 \\
\hline 13 & 97.6 & 7.9 & 72 & 4 & 13.5 & 6.7 \\
\hline 14 & 48.4 & 6.4 & 61 & 2 & 10.5 & 7.9 \\
\hline 15 & 241.8 & 11.3 & 35 & 13 & 11.8 & 5.1 \\
\hline 16 & 89.2 & 5.6 & 54 & 10 & 14.0 & 5.3 \\
\hline 17 & 122.5 & 8.0 & 79 & 6 & 8.9 & 6.0 \\
\hline 18 & 139.1 & 4.9 & 68 & 9 & 13.6 & 3.8 \\
\hline 19 & 107.6 & 7.3 & 116 & 3 & 13.0 & 5.4 \\
\hline 20 & 118.3 & 8.4 & 84 & 11 & 10.6 & 5.2 \\
\hline 21 & 261.2 & 6.6 & 67 & 13 & 11.2 & 6.4 \\
\hline 22 & 79.5 & 4.7 & 46 & 7 & 8.5 & 4.8 \\
\hline
\end{tabular}



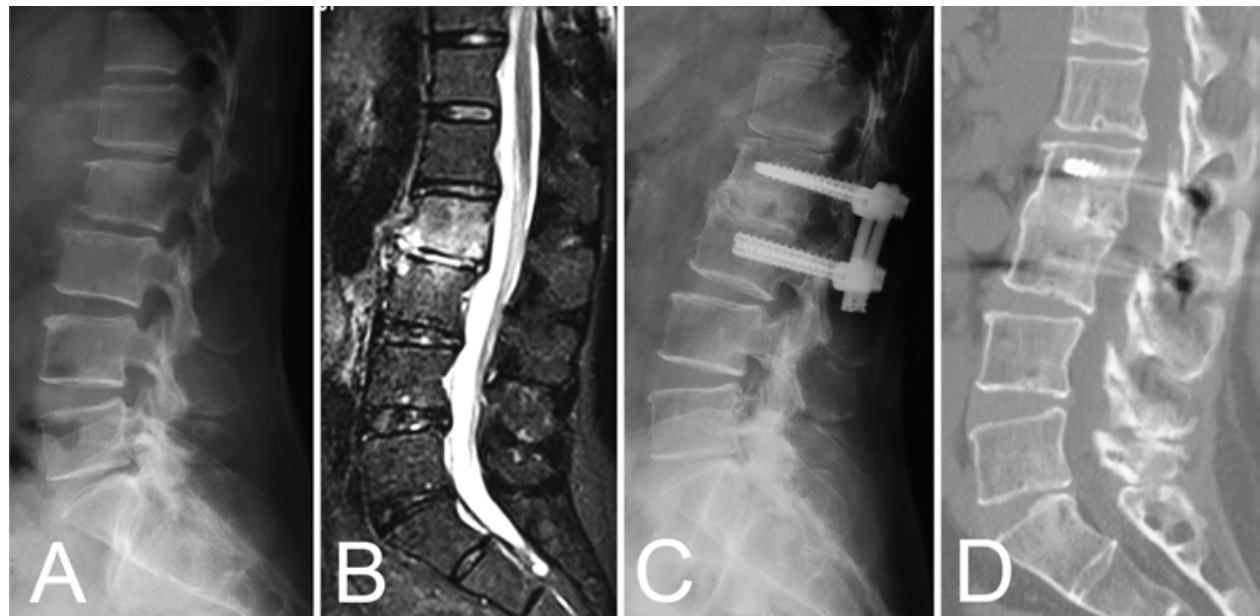

FIG. 2. Preoperative and postoperative radiological images in a patient. Preoperative radiograph (A) and MRI (B) showing pyogenic spondylodiscitis at the L2-3 level. Postoperative radiograph (C) and CT (D) showing good intervertebral bony fusion.

and severe intraoperative blood loss. In the present study, we performed mini-open anterior debridement and lumbar interbody fusion in combination with posterior percutaneous fixation for the treatment of single-level lumbar pyogenic spondylodiscitis. Compared with minimally invasive posterior procedures such as transforaminal lumbar interbody fusion and posterior lumbar interbody fusion, this approach allows the thorough removal of infective lesions and prevents bacterial spread to the instruments and muscles posterior to the vertebrae.

The conventional posterior midline approach involves extensive dissection and retraction of the paraspinal mus-

TABLE 3. Clinical outcomes of 22 patients with lumbar pyogenic spondylodiscitis

\begin{tabular}{|c|c|c|c|c|c|c|c|c|c|}
\hline \multirow[b]{2}{*}{ Case No. } & \multirow{2}{*}{$\begin{array}{c}\text { Follow-Up } \\
\text { (mos) }\end{array}$} & \multicolumn{2}{|c|}{ ASIA Grade } & \multicolumn{2}{|c|}{ JOA Score } & \multicolumn{2}{|c|}{ VAS Score } & \multicolumn{2}{|c|}{ ODI (\%) } \\
\hline & & Preop & Final Follow-Up & Preop & Final Follow-Up & Preop & Final Follow-Up & Preop & Final Follow-Up \\
\hline 1 & 37 & $E$ & E & 10 & 27 & 8 & 1 & 88 & 14 \\
\hline 2 & 32 & E & $E$ & 9 & 27 & 7 & 0 & 80 & 20 \\
\hline 3 & 25 & D & $E$ & 6 & 28 & 7 & 1 & 78 & 8 \\
\hline 4 & 31 & E & E & 8 & 27 & 7 & 0 & 86 & 16 \\
\hline 5 & 35 & C & D & 3 & 23 & 8 & 0 & 72 & 16 \\
\hline 6 & 29 & D & $\mathrm{E}$ & 8 & 28 & 8 & 1 & 78 & 6 \\
\hline 7 & 24 & $E$ & $E$ & 10 & 25 & 7 & 0 & 74 & 24 \\
\hline 8 & 30 & D & $E$ & 6 & 27 & 7 & 0 & 92 & 10 \\
\hline 9 & 32 & $E$ & $E$ & 11 & 28 & 7 & 1 & 82 & 8 \\
\hline 10 & 25 & $\mathrm{E}$ & $E$ & 10 & 25 & 8 & 1 & 80 & 12 \\
\hline 11 & 27 & $\mathrm{E}$ & $\mathrm{E}$ & 10 & 26 & 9 & 0 & 76 & 10 \\
\hline 12 & 28 & $\mathrm{E}$ & $E$ & 13 & 25 & 6 & 1 & 86 & 16 \\
\hline 13 & 32 & D & $E$ & 10 & 24 & 7 & 1 & 84 & 26 \\
\hline 14 & 30 & $E$ & $E$ & 12 & 28 & 6 & 0 & 78 & 8 \\
\hline 15 & 28 & D & D & 7 & 26 & 8 & 0 & 88 & 12 \\
\hline 16 & 28 & $E$ & $E$ & 9 & 26 & 7 & 0 & 90 & 16 \\
\hline 17 & 33 & D & $\mathrm{E}$ & 9 & 27 & 8 & 1 & 80 & 8 \\
\hline 18 & 35 & $\mathrm{E}$ & $\mathrm{E}$ & 11 & 23 & 7 & 2 & 76 & 22 \\
\hline 19 & 38 & $\mathrm{E}$ & $E$ & 10 & 25 & 8 & 1 & 82 & 18 \\
\hline 20 & 37 & D & $\mathrm{E}$ & 8 & 25 & 7 & 0 & 74 & 20 \\
\hline 21 & 29 & $E$ & $\mathrm{E}$ & 9 & 26 & 9 & 1 & 90 & 14 \\
\hline 22 & 31 & $\mathrm{E}$ & $E$ & 11 & 24 & 8 & 2 & 78 & 20 \\
\hline Mean \pm SD & & & & $9.1 \pm 2.2$ & $25.9 \pm 1.6^{*}$ & $7.5 \pm 0.8$ & $0.6 \pm 0.7^{\star}$ & $81.5 \pm 5.8$ & $14.7 \pm 5.7^{*}$ \\
\hline
\end{tabular}

${ }^{*} p<0.001$ compared with preoperative values. 
cles, leading to postoperative back pain, reduced lumbar muscle strength, and slow recovery time. ${ }^{24}$ The Wiltse approach is another choice, but still causes a certain amount of muscle trauma and skin incision..$^{15}$ In the present study, we used posterior percutaneous pedicle screw fixation, which has been reported to be an effective and safe method for the treatment of thoracic and lumbar burst fracture..$^{18}$ Under the guidance of preoperative imaging and intraoperative fluoroscopy, percutaneous pedicle screw fixation can be effectively performed with the appropriate type of screws and the correct angle, and thus can produce fixation similar to that of conventional open surgery. The advantages of this approach over open surgery include minimizing intraoperative retraction of the paraspinal muscles and ligaments, shortening the surgical time, reducing intraoperative blood loss, and promoting the postoperative recovery of lumbar muscle strength. ${ }^{10}$

Conventional anterior open surgery can achieve good debridement and bone implantation, ${ }^{8,21,22}$ but often results in an incision longer than $15 \mathrm{~cm}$, extensive dissection of the muscles, severe blood loss, and a high risk of injury to the major blood vessels, ureter, lumbar plexus, and peritoneum. In the present study, we adopted the mini-open modified ALIF ${ }^{16}$ approach by establishing a working channel directly to the surgical area, which was about $5 \mathrm{~mm}$ behind the anterior edge of the psoas major muscle. This opens a window onto the psoas major muscle, through which debridement could be expanded and fusion performed in the intervertebral space.

Our approach reduced the risk of injury to the major blood vessels and sympathetic trunk, which is associated with performing conventional ALIF in front of the psoas major muscle. In fact, for pyogenic spondylodiscitis, the traditional ALIF approach is difficult, and it is dangerous to separate the tissues between the psoas major muscle and the major blood vessels because the normal tissue planes are often obscured by scar formation and the result is chronic infection. It is much safer to pass through the psoas major muscle.

Compared with extreme lateral interbody fusion, ${ }^{20}$ our approach reduces retraction of the psoas major muscle and lumbar plexus. ${ }^{1}$ Because debridement is performed anterior to the paravertebral abscess, the lumbar plexus is pushed backward by the abscess, far away from the debridement area. Once the working channel is established, it is sufficient and fixed for complete debridement and bone transplantation, thus reducing the risk of injury to the peritoneum and its surrounding structures. In addition, the pressure from the automatic retractor on the muscles reduces muscle bleeding.

The anterior approach that passes the psoas major muscle carries a high risk of iatrogenic injury to the lumbar plexus, ${ }^{2}$ which is caused by direct mechanical compression, overstretching, tearing, electric cautery, and ischemia. Numbness in the lower abdomen, groin, and thigh and weakness in the hip flexor and knee extensor can occur postoperatively. Another complication associated with the anterior approach is injury to the blood vessels, which can result in severe consequences. To avoid these complications, care should be taken with regard to the following: 1) The left lateral approach should be taken to reduce the risk of injury to the inferior vena cava, except for cases with a huge abscess on the right. 2) To facilitate surgery, the patient's position should be adjusted preoperatively under fluoroscopic control to ensure that the targeted intervertebral space is perpendicular to the operating table. 3) Muscles should be dissected along the direction of their fiber orientation. 4) Electric cautery should be avoided, and care should be taken to avoid injury to the lumbar plexus and sympathetic chains. 5) The ureter should not be exposed, and can be pushed forward with the retroperitoneal fat tissues. 6) Care should be taken to avoid injury to the genitofemoral nerve. 7) The common iliac vessels should be protected at the L4-5 level. 8) Thorough debridement is important to achieving a good therapeutic outcome. In the present study, transient urinary retention occurred in 5 (23\%) of 22 cases. Urinary retention was transient and recovered after symptomatic treatment without sequelae. Transient urinary retention may result from the use of patient-controlled analgesia, Foley catheterization, incision pain, or prolonged bed rest.

This combined mini-open approach requires surgeons to have enough experience with open surgery. Surgeons should be proficient with the anatomy and dissect several muscular layers directly to the surgical area. Beginners may encounter difficulties in exposing the surgical field. Precise preoperative surface location is important for determining the position and size of the incision. If the incision site deviates too much, the affected segment may not be exposed sufficiently, thus leading to the failure of the mini-open approach and conversion to open surgery. To reduce errors, multiple intraoperative fluoroscopic examinations should be performed to guide the surgical procedure. However, multiple fluoroscopic examinations can lead to an increase in x-ray exposure for the patient and surgeons. In addition, this method is only indicated for patients with single-segment lumbar pyogenic spondylodiscitis, or certain patients with 2-segment involvement. In the present study, only patients with single-segment lumbar pyogenic spondylodiscitis were included. For patients with involvement of the L5-S1 segment, this mini-open approach is not suitable because it is hindered by the ilium and thus exposure is difficult.

\section{Conclusions}

Mini-open anterior debridement and lumbar interbody fusion in combination with posterior percutaneous fixation resulted in little surgical trauma and intraoperative blood loss and few postoperative complications, suggesting that this approach is effective and safe for the treatment of single-level lumbar pyogenic spondylodiscitis. This approach could be an alternative to the conventional open surgery.

\section{References}

1. Aghayev K, Vrionis FD: Mini-open lateral retroperitoneal lumbar spine approach using psoas muscle retraction technique. Technical report and initial results on six patients. Eur Spine J 22:2113-2119, 2013

2. Ahmadian A, Deukmedjian AR, Abel N, Dakwar E, Uribe JS: Analysis of lumbar plexopathies and nerve injury after lateral retroperitoneal transpsoas approach: diagnostic standardization. J Neurosurg Spine 18:289-297, 2013 
3. Bettini N, Girardo M, Dema E, Cervellati S: Evaluation of conservative treatment of non specific spondylodiscitis. Eur Spine J 18 (Suppl 1):143-150, 2009

4. Cebrián Parra JL, Saez-Arenillas Martín A, Urda MartínezAedo AL, Soler Ivañez I, Agreda E, Lopez-Duran Stern L: Management of infectious discitis. Outcome in one hundred and eight patients in a university hospital. Int Orthop 36:239-244, 2012

5. Fairbank J: Use of Oswestry Disability Index (ODI). Spine (Phila Pa 1976) 20:1535-1537, 1995

6. Ford LT: Postoperative infection of lumbar intervertebral disk space. South Med J 69:1477-1481, 1976

7. Friedman JA, Maher CO, Quast LM, McClelland RL, Ebersold MJ: Spontaneous disc space infections in adults. Surg Neurol 57:81-86, 2002

8. Gonzalvo A, Abdulla I, Riazi A, De La Harpe D: Singlelevel/single-stage debridement and posterior instrumented fusion in the treatment of spontaneous pyogenic osteomyelitis/discitis: long-term functional outcome and health-related quality of life. J Spinal Disord Tech 24:110-115, 2011

9. Hoelscher GL, Gruber HE, Coldham G, Grigsby JH, Hanley EN Jr: Effects of very high antibiotic concentrations on human intervertebral disc cell proliferation, viability, and metabolism in vitro. Spine (Phila Pa 1976) 25:1871-1877, 2000

10. Kim DY, Lee SH, Chung SK, Lee HY: Comparison of multifidus muscle atrophy and trunk extension muscle strength: percutaneous versus open pedicle screw fixation. Spine (Phila Pa 1976) 30:123-129, 2005

11. Kirshblum SC, Burns SP, Biering-Sorensen F, Donovan W, Graves DE, Jha A, et al: International standards for neurological classification of spinal cord injury (revised 2011). J Spinal Cord Med 34:535-546, 2011

12. Knop C, Oeser M, Bastian L, Lange U, Zdichavsky M, Blauth M: [Development and validation of the Visual Analogue Scale (VAS) Spine Score.] Unfallchirurg 104:488-497, 2001 (Ger)

13. Ledermann HP, Schweitzer ME, Morrison WB, Carrino JA: MR imaging findings in spinal infections: rules or myths? Radiology 228:506-514, 2003

14. Lee JS, Suh KT: Posterior lumbar interbody fusion with an autogenous iliac crest bone graft in the treatment of pyogenic spondylodiscitis. J Bone Joint Surg Br 88:765-770, 2006

15. Lin Y, Chen WJ, Zhu WT, Li F, Fang H, Chen AM, et al: Single-level lumbar pyogenic spondylodiscitis treated with minimally invasive anterior debridement and fusion combined with posterior fixation via Wiltse approach. J Huazhong Univ Sci Technolog Med Sci 33:707-712, 2013

16. Mayer HM: A new microsurgical technique for minimally invasive anterior lumbar interbody fusion. Spine (Phila Pa 1976) 22:691-700, 1997

17. McHenry MC, Easley KA, Locker GA: Vertebral osteomyelitis: long-term outcome for 253 patients from 7 Clevelandarea hospitals. Clin Infect Dis 34:1342-1350, 2002
18. Ni WF, Huang YX, Chi YL, Xu HZ, Lin Y, Wang XY, et al: Percutaneous pedicle screw fixation for neurologic intact thoracolumbar burst fractures. J Spinal Disord Tech 23:530-537, 2010

19. Osawa T, Ogura T, Hayashida T, Mori M, Hase H: Evaluation of lumbosacral nerve root lesions using evoked potentials recorded by a surface electrode technique. Spine (Phila Pa 1976) 28:496-501, 2003

20. Ozgur BM, Aryan HE, Pimenta L, Taylor WR: Extreme Lateral Interbody Fusion (XLIF): a novel surgical technique for anterior lumbar interbody fusion. Spine J 6:435-443, 2006

21. Pee YH, Park JD, Choi YG, Lee SH: Anterior debridement and fusion followed by posterior pedicle screw fixation in pyogenic spondylodiscitis: autologous iliac bone strut versus cage. J Neurosurg Spine 8:405-412, 2008

22. Sundararaj GD, Babu N, Amritanand R, Venkatesh K, Nithyananth M, Cherian VM, et al: Treatment of haematogenous pyogenic vertebral osteomyelitis by single-stage anterior debridement, grafting of the defect and posterior instrumentation. J Bone Joint Surg Br 89:1201-1205, 2007

23. Tyler KL: Acute pyogenic diskitis (spondylodiskitis) in adults. Rev Neurol Dis 5:8-13, 2008

24. Vialle R, Wicart P, Drain O, Dubousset J, Court C: The Wiltse paraspinal approach to the lumbar spine revisited: an anatomic study. Clin Orthop Relat Res 445:175-180, 2006

25. Wang X, Zhou J, Zhang C, Liu Z: Single-stage anterior debridement and fusion with autografting and internal fixation for pyogenic lumbar spondylodiscitis. Arch Orthop Trauma Surg 132:487-493, 2012

\section{Disclosure}

The authors report no conflict of interest concerning the materials or methods used in this study or the findings specified in this paper.

\section{Author Contributions}

Conception and design: Xiong, Lin, Li. Acquisition of data: Xiong, Lin. Analysis and interpretation of data: Lin, W Chen. Drafting the article: Lin, W Chen. Critically revising the article: Xiong, Lin. Reviewed submitted version of manuscript: Xiong, Lin. Approved the final version of the manuscript on behalf of all authors: Xiong. Statistical analysis: Lin. Administrative/technical/ material support: Xiong, Li, Zeng, A Chen. Study supervision: Xiong, Li, A Chen.

\section{Correspondence}

Wei Xiong, Department of Orthopedics, Tongji Hospital of Tongji Medical College, Huazhong University of Science and Technology, 1095 Jie Fang Ave., Wuhan 430030, P. R. China. email:xcxgreatwellus@hotmail.com. 